\title{
Effects of a resistance training program on muscular performance adaptations: comparing three vs. four times per week
}

\author{
Hossein Firoozi ${ }^{1}$, Hamid Arazi $^{1}$, Abbas Asadi $^{2}$ \\ 1 Department of Exercise Physiology, Faculty of Sport Sciences, University of Guilan, Rasht, Iran; ${ }^{2}$ Department of \\ Physical Education and Sport Sciences, Payame Noor University, Tehran, Iran
}

\section{Summary}

Study aim: The aim of this study was to examine the effects of 8 weeks of resistance training (RT) with three vs. four sessions per week and equated training volume on muscular adaptations in men.

Materials and methods: Thirty-three healthy young men volunteered to participate in the study and were randomly assigned to three times per week whole-body RT (RT3, $n=11)$, four times per week whole-body RT (RT4, $\mathrm{n}=11$ ) or a control group $(\mathrm{CG}, \mathrm{n}=11)$. Before and after training, participants were evaluated for one-repetition maximum (1RM) and muscular endurance (i.e., $60 \%$ of 1RM to failure) for the leg press and bench press. In addition, thigh, arm, chest, and calf circumferences, and percent body fat were assessed before and after training.

Results: The findings revealed significant main effects of time for chest and thigh circumferences $(\mathrm{p} \leq 0.05)$. There were no significant group $\times$ time interactions for chest and thigh circumferences $(\mathrm{p}>0.05)$, but the RT4 showed greater changes (effect size [ES]: 0.48 vs. 0.15 ) in chest circumference, while the RT3 showed greater changes (ES: 0.77 vs. 0.35$)$ in thigh circumference. Significant group $\times$ time interactions were observed for the 1 RM of leg and bench presses $(p<0.05)$. Post-hoc analyses showed greater improvements for RT3 in comparison to RT4 in 1RM bench press ( $\mathrm{p}=0.01$, ES: 0.77 vs. 0.6) and leg presses $(\mathrm{p}=0.009$, ES: 0.94 vs. 0.86).

Conclusions: These results suggest that RT induces meaningful adaptive effects to improve strength and muscle size in men and RT3 appears to be more effective to induce muscular adaptations.

Keywords: Frequency - Strength training - Body composition - Performance - Workout

\section{Introduction}

It has been well documented that resistance training (RT) is an optimum training modality to improve strength, power and hypertrophy $[2,7,11]$. The design of optimum RT programs requires the control of several variables including exercise selection, exercise order, training load and repetitions, rest interval between sets and training frequency [11]. Regarding the latter, some studies recently focused on the effects of training frequency on muscular adaptations [4, 6, 7]. To date, frequency defined as the number of RT sessions which performed by the athletes for per muscle group during a week [9]. The American College of Sports Medicine reported that 2 or 3 sessions per week RT using a split or total body routine could be an optimal dose for enhancing performance adaptations in novice and intermediately trained individuals [2]. In general, RT programs for enhancing muscle hypertrophy are performed with a high volume of work per muscle group in a session $[1,7]$; however, others suggest that split routine training per muscle group allows greater recovery between sessions and helps to increase muscle size [13, 14, 20, 21].

Schoenfeld et al. [23, 24] performed some meta-analyses on the effects of RT frequency on muscular strength and hypertrophy and stated that two sessions per week are more effective than one session per week to enhance muscle hypertrophy $[13,14,23,24]$. In contrast, during equated RT volume no significant differences were observed in muscular strength and hypertrophy by different training frequencies $[23,24]$.

In experimental studies, a large number of studies compared one vs. two and three, three vs. four and six days per week RT on muscular adaptations and the results are controversial $[4,6,7,12,15,16,20,22,25,26]$; 
however, it seems that three days per week RT could be an optimum training modality for adaptations. Furthermore, almost all the studies examined training for three days per week or less; none of the included studies investigated high training days such as RT performed four days per week and did not compare split routine RT for three vs. four days per week on muscular adaptations, and data about this subject are unknown. In fact, for gains in strength, the current findings indicate that similar strength gains can be attained using vastly different training frequencies using split routine training, provided that total volume is equated [14] and the responses to high frequencies such as training 4 times per week are still being investigated.

Therefore, taking into account the evident lack of similar studies conducted in this area, the purpose of this study was to examine the effects of 8 weeks of equal-volume RT differing in frequency (3 vs. 4 days) on muscular strength, endurance, and hypertrophy in young men. We hypothesized that 1) 8-week RT would increase muscular adaptations in men and 2) both RT frequencies would show similar improvements in analyzed outcomes due to training volume being equaled.

\section{Materials and methods}

\section{Participants}

Thirty-three healthy young men who were familiar with resistance exercise and training volunteered to participate in this study and were randomly assigned to three times per week whole-body RT $($ RT3; $n=11$ age $=21.9 \pm 1.3 \mathrm{y}$, height $=178.7 \pm 9.3 \mathrm{~cm}$, weight $=68.1 \pm 4.1 \mathrm{~kg}$, and RT experience $3.2 \pm 0.9$ years) or four times per week whole-body RT $(\mathrm{RT} 4 ; \mathrm{n}=11$ age $=21.0 \pm 1.0 \mathrm{y}$, height $=$ $178.0 \pm 5.9 \mathrm{~cm}$, weight $=66.3 \pm 7.4 \mathrm{~kg}$, and RT experience $3.5 \pm 1.1$ years) and a control group of subjects who did not perform any RT program throughout the study period $(\mathrm{CG} ; \mathrm{n}=11$, age $=22.4 \pm 1.3 \mathrm{y}$, height $=175.2 \pm 6.2 \mathrm{~cm}$, weight $=68.8 \pm 7.6 \mathrm{~kg}$, and RT experience $3 \pm 0.8$ years) . A prior estimated sample size for $\mathrm{b}=0.80$ with $\mathrm{a}=0.05$ was calculated based on data from a previous research study [10]. The inclusion criteria for this study included: 1) no history of medical or orthopedic problems that compromised their participation in this study, 2) no lower extremity reconstructive surgery of any kind in the past two years or unresolved musculoskeletal disorders, and 3) no use of drugs or supplements throughout 6 months before initiation of the study. Each subject was informed of the risks and benefits of the study and subsequently signed an informed consent form in accordance with the guidelines of the university's Institutional Review Board. The procedures were approved by the Research Committee of the University (DT-139700).

\section{Study design}

This study followed a randomized, controlled, longitudinal design. Subjects were pair matched according to demographic variables and then randomly divided into 2 experimental groups - RT3, where resistance exercises were performed on 3 days per week, and RT4, where resistance exercises were performed on 4 days per week - and a control group. The time of training, range of motion, rest interval between sets and exercises and also training volume were the same in both training groups. The study period lasted 11 weeks as follows: $1^{\text {st }}$ week - familiarization period; $2^{\text {nd }}$ week - pretest period; $3^{\text {rd }}$ to $10^{\text {th }}$ week - training period; $11^{\text {th }}$ week - post-test period. The training intervention period lasted 8 weeks and the subjects performed equal volume training loads and exercises with different training days. Testing was performed before and after the training period for measuring body weight, body fat, chest, arm, thigh and calf circumferences, 1 repetition maximum (1RM) and muscular endurance for the barbell bench press exercise and machine leg press exercise. The test-retest reliability coefficient of these tests was $r \geq 0.95$. To avoid potential dietary confounding of results, subjects were advised to maintain their customary nutritional regimen and to avoid taking any supplements during the study period.

\section{Procedures}

The subjects underwent 4 days of testing, namely 2 pre- (72 h apart between testing sessions) and 2 post-test days ( $72 \mathrm{~h}$ apart between testing sessions). The subjects were tested at the exact same time of day ( 2 to 4 p.m., post-test day) and the same day of the week as the pre-test days to minimize the effect of circadian variations in the test results. All subjects had to continue with the normal daily life activity and dietary intake.

\section{Anthropometric assessment}

Height was assessed using a wall-mounted stadiometer (Seca 222, Terre Haute, IN) recorded to the nearest $0.1 \mathrm{~cm}$. Body mass was measured to the nearest $0.1 \mathrm{~kg}$ using a medical scale (Tanita, BC-418MA, Tokyo, Japan). To determine the percent body fat, skinfold thickness was measured (Lafayette Caliper, model 01128, USA) at 9 sites (i.e., chest, abdominal, thigh, biceps, triceps, subscapular, suprailiac, lower back, and calf) on the right side of the body during standing in accordance with the Parillo method using the following formula: \% body fat $=$ (sum of 9 sites $\times 27$ ) divided by bodyweight (lbs) [18]. Each site measurement was assessed 3 times and the average of 3 trials was recorded for analysis. The circumferences of chest, mid-thigh, mid-arm and mid-calf of the right side were measured to the nearest $0.1 \mathrm{~cm}$ for determining muscle size changes following 8 weeks of RT [5]. All anthropometric measures were assessed by the same researcher who was experienced and qualified for the measurements. 


\section{Muscular strength assessment}

Upper-body strength was assessed first, using the barbell bench press exercise; lower body strength was assessed second, using a $45^{\circ}$ leg press machine (Nebula Fitness, Inc., Versailles, OH) [5, 25]. To determine 1RM, participants performed 8 to 10 repetitions using $\sim 50 \%$ of $1 \mathrm{RM}$ for warm up, 3 to 5 repetitions using $\sim 75 \%$ of $1 \mathrm{RM}$, and 1 to 3 repetitions using $\sim 90 \%$ of $1 \mathrm{RM}$. After 3 trials, the intensity of resistance was increased until the subjects were unable to complete an attempt using proper technique through a full range of motion. At least 5 maximal trials were allowed, with 5 minutes of rest between attempts to evaluate 1RM. A Certificated Strength and Conditioning Specialist supported the subjects during 1RM testing for encouragement and to ensure safety.

\section{Muscular endurance assessment}

The muscular endurance test (i.e., bench press and leg press exercises) was conducted $72 \mathrm{~h}$ after maximal strength tests. After a short period of warm-up (i.e., $5 \mathrm{~min}$ of running and $5 \mathrm{~min}$ of stretching exercise), participants performed 10 repetitions (i.e., $30 \%$ of 1RM for warm-up) of each exercise test and then performed as many as possible repetitions to exhaustion without stopping or pausing between repetitions. The resistance intensity was selected as $60 \%$ of $1 \mathrm{RM}$, as recommended previously for assessing muscular endurance [3].

\section{Training protocol}

A summary of the RT programs can be found in Table 1 . The training protocol included a mixture of single-joint and multi-joint exercises which was designed in differing training session days as bodybuilders perform training to improve muscle hypertrophy and adaptation. The subjects in the CG did not participate in any RT program and continued their habitual daily life activities. Each training session lasted 80 and 65 min including $10 \mathrm{~min}$ warm-up, 60 and 45 min main training for the RT3 and RT4, respectively, and 10 min cool-down; however, weekly training set, load, volume and duration were equated between the training groups. All exercises were performed for 3 sets of 8 repetitions with 60 to $90 \mathrm{~s}$ of rest between sets and 2 to $3 \mathrm{~min}$ of rest between exercises. The intensity of exercise included $65 \%$ of $1 \mathrm{RM}$ in weeks 1 and $2,70 \%$ of $1 \mathrm{RM}$ in weeks 3 and $4,75 \%$ of $1 \mathrm{RM}$ in weeks 5 and 6 , and $80 \%$ of 1RM in weeks 7 and 8 for both the training groups [11]. Each training session was supervised by a researcher and Certificated Strength and Conditioning Specialist.

\section{Statistical analyses}

All data are presented as mean \pm SD. The Shapiro-Wilk test was used to determine whether the data were normally distributed. A two-way analysis of variance with repeated measures $(3$ [group] $\times 2$ [time] $)$ was used to determine significant differences among groups. When a significant $F$ value was achieved, Bonferroni post hoc procedures were performed to identify the pairwise differences between the values. The level of significance was set at $\mathrm{P} \leq 0.05$. Effect sizes (ES) were calculated using Cohen's $d$. Threshold values for assessing magnitudes of ES were $<0.2$, trivial; $0.2-0.6$, small; $0.6-1.2$, moderate; $1.2-2.0$, large; $2.0-4.0$, very large; and $>4.0$, nearly perfect [17] with the $95 \%$ confidence interval (CI). In addition, relative strength for 1RM bench press and leg press were calculated by dividing absolute load (i.e., 1RM weight) by subjects' body weight.

\section{Results}

Before training, there were no significant differences among the groups RT3, RT4 and CG in dependent variables $(p>0.05)$ (Table 2). No significant changes in the CG were observed in all variables after the training period $(p>0.05)$. In addition, the RT3 and RT4 indicated significant differences in comparison to the CG in 1RM bench press and leg press following the 8 -week training intervention $(\mathrm{p}<0.05)$.

\section{Anthropometric}

There were no significant main effects of time $(\mathrm{F}=2.8$, $\mathrm{p}=0.11)$ or group $\times$ time $(\mathrm{F}=4.2, \mathrm{p}=0.11)$ interactions for weight; however, the RT3 $(\mathrm{ES}=0.31)$ and RT4 $(\mathrm{ES}=0.11)$ groups showed small and trivial ES in weight after the training intervention (Table 2).

In percent body fat, both the RT3 and RT4 groups showed trivial $(\mathrm{ES}=-0.18)$ to small $(\mathrm{ES}=-0.5)$ decreases after 8 weeks of training, but these changes were not statistically significant in main effects of time $(\mathrm{F}=3.1, \mathrm{p}=0.1)$ or group $\times$ time $(F=2.06, p=0.16)$ interactions (Table 2$)$.

Significant main effects of time $(F=11.9, p=0.006$, $\mathrm{F}=4.1, \mathrm{p}=0.05)$, but not group $\times$ time $(\mathrm{F}=2.4, \mathrm{p}=0.12$, $\mathrm{F}=1.8, \mathrm{p}=0.2)$ interactions were observed for chest and thigh circumferences, respectively. In addition, the RT3 group showed a trivial to moderate $(\mathrm{ES}=0.15$ and 0.77$)$ ES, while the RT4 group showed a small (ES $=0.48$ and 0.35 ) ES in chest and thigh circumferences, respectively (Table 2).

There were no significant main effects of time $(\mathrm{F}=0.01$, $\mathrm{p}=0.9, \mathrm{~F}=1.3, \mathrm{p}=0.16)$ or group $\times$ time $(\mathrm{F}=0.57$, $\mathrm{p}=0.47, \mathrm{~F}=1.8, \mathrm{p}=0.2)$ interactions for arm and calf circumferences, respectively. In addition, the RT3 group showed a trivial to small ( $\mathrm{ES}=0.17$ and 0.21$) \mathrm{ES}$, while the RT4 group showed a moderate ( $\mathrm{ES}=0.44$ and 0.44$) \mathrm{ES}$ in arm and calf circumferences, respectively (Table 2).

\section{Strength and endurance}

There was a significant main effect of time $(\mathrm{F}=58.6$, $\mathrm{p}=0.001)$ and group $\times$ time $(\mathrm{F}=7.4, \mathrm{p}=0.01)$ interaction 


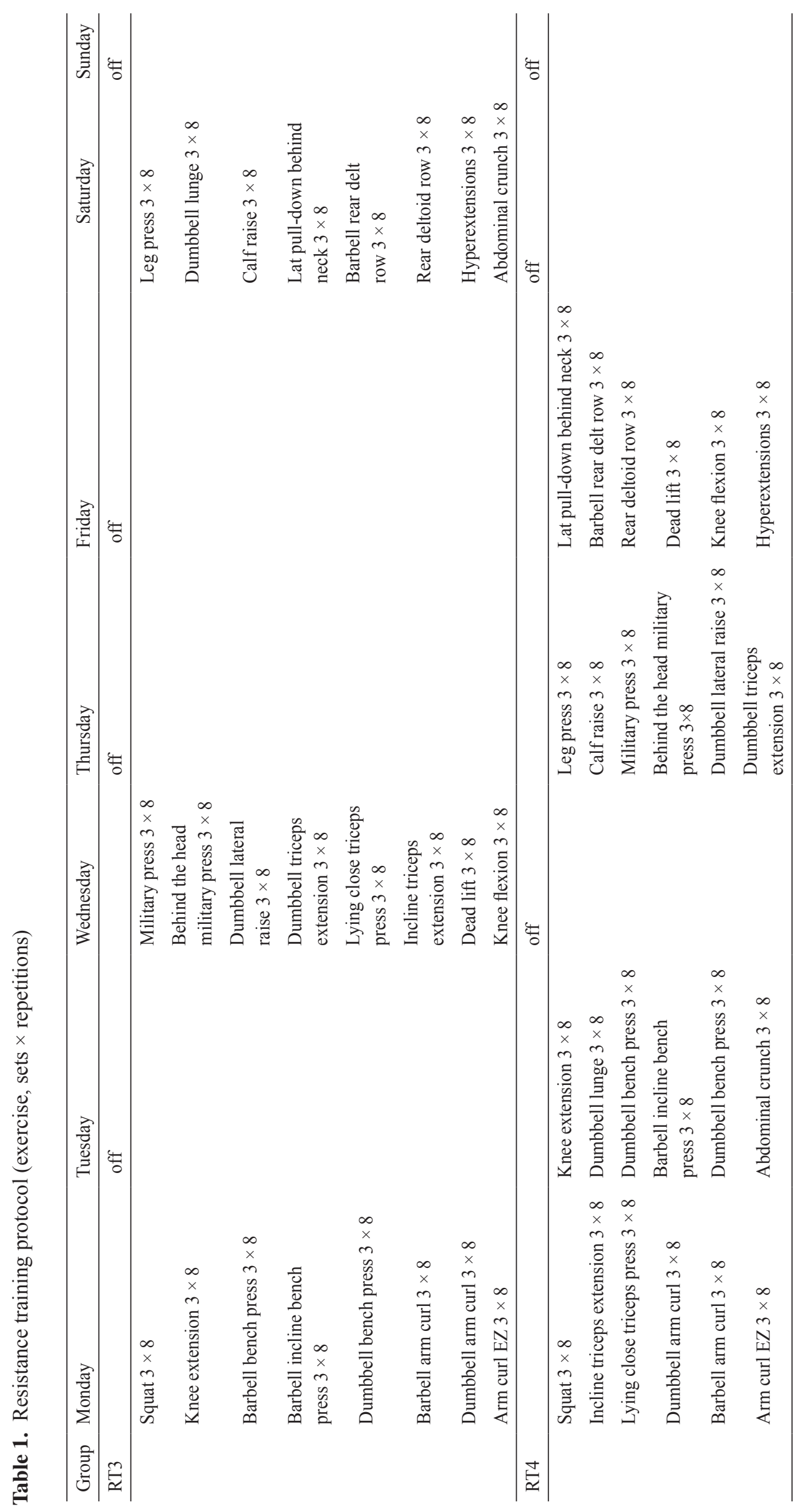


Table 2. Changes in anthropometric and performance variables in response to 8 weeks training intervention (mean $\pm \mathrm{SD})$

\begin{tabular}{lllll}
\hline Variables & RT3 $(\mathrm{n}=11)$ & RT4 $(\mathrm{n}=11)$ & CG $(\mathrm{n}=11)$ & Significance $(\mathrm{p}$-values $)$ \\
\hline Weight $[\mathrm{kg}]$ & & & & \\
\hline Before & $68.1 \pm 4.1$ & $66.3 \pm 7.4$ & $68.8 \pm 7.6$ & G: 0.08 \\
After & $69.4 \pm 4.2$ & $67.0 \pm 5.0$ & $68.5 \pm 7.2$ & T: 0.11 \\
ES & $0.31(-0.54$ to 1.14$) \mathrm{b}$ & $0.11(-0.73$ to 0.94$) \mathrm{a}$ & $0.04(-0.8$ to 0.87$)$ & $\mathrm{G} \times \mathrm{T}: 0.06$ \\
\hline Body fat $[\%]$ & & & $13.6 \pm 3.2$ & G: 0.54 \\
\hline Before & $13.0 \pm 2.8$ & $14.3 \pm 1.6$ & $13.8 \pm 3.3$ & T: 0.1 \\
After & $12.5 \pm 2.9$ & $13.5 \pm 1.6$ & G $\times$ T: 0.16 \\
ES & $-0.18(-1.01$ to 0.67$) \mathrm{a}$ & $-0.5(-1.33$ to 0.37$) \mathrm{b}$ & $0.06(-0.78$ to 0.9$)$ & \\
\hline
\end{tabular}

Chest circumference $[\mathrm{cm}]$

\begin{tabular}{lllll}
\hline Before & $96.5 \pm 9.5$ & $88.5 \pm 4.7$ & $89.8 \pm 5.6$ & G: 0.1 \\
After & $97.9 \pm 9.1^{*}$ & $90.8 \pm 4.8^{*}$ & $90.3 \pm 6.0$ & T: 0.006 \\
ES & $0.15(-0.69$ to 0.98$)$ a & $0.48(-0.38$ to 1.31$)$ b & $0.09(-0.75$ to 0.92$)$ & G $\times$ T: 0.12 \\
\hline
\end{tabular}

Thigh circumference $[\mathrm{cm}]$

\begin{tabular}{lllll}
\hline Before & $52.6 \pm 4.2$ & $50.2 \pm 3.3$ & $48.4 \pm 4.3$ & G: 0.12 \\
After & $55.5 \pm 3.3^{*}$ & $51.4 \pm 3.5^{*}$ & $48.9 \pm 4.5$ & T: 0.05 \\
ES & $0.77(-0.13$ to 1.6$) \mathrm{c}$ & $0.35(-0.50$ to 1.18$) \mathrm{b}$ & $0.11(-0.73$ to 0.95$)$ & $\mathrm{G} \times \mathrm{T}: 0.2$ \\
\hline Arm circumference $[\mathrm{cm}]$ & & & \\
\hline Before & $29.8 \pm 2.4$ & $27.3 \pm 2.2$ & $28.0 \pm 1.9$ & G: 0.48 \\
After & $28.8 \pm 8.1$ & $28.5 \pm 3.2$ & $28.1 \pm 2.0$ & T: 0.9 \\
ES & $-0.17(-1.0$ to 0.68$) \mathrm{a}$ & $0.44(-0.42$ to 1.27$) \mathrm{b}$ & $0.05(-0.79$ to 0.89$)$ & $\mathrm{G} \times \mathrm{T}: 0.47$ \\
\hline
\end{tabular}

Calf circumference $[\mathrm{cm}]$

\begin{tabular}{lllll}
\hline Before & $37.3 \pm 2.4$ & $35.1 \pm 1.9$ & $36.0 \pm 2.6$ & G: 0.1 \\
After & $37.8 \pm 2.3$ & $36.0 \pm 2.2$ & $36.2 \pm 2.3$ & T: 0.16 \\
ES & $0.21(-0.63$ to 1.04$) \mathrm{b}$ & $0.44(-0.42$ to 1.27$) \mathrm{b}$ & $0.08(-0.76$ to 0.91$)$ & $\mathrm{G} \times \mathrm{T}: 0.20$ \\
\hline
\end{tabular}

$1 \mathrm{RM}$ bench press $[\mathrm{kg}]$

\begin{tabular}{lllll}
\hline Before & $78.5 \pm 8.8$ & $70.8 \pm 10.9$ & $73.1 \pm 12.9$ & G: 0.11 \\
After & $85.9 \pm 10.3^{* * * \dagger}$ & $77.7 \pm 11.5^{* \dagger}$ & $73.7 \pm 13.4$ & T: 0.001 \\
ES & $0.77(-0.12$ to 1.61$) \mathrm{c}$ & $0.6(-0.26$ to 1.45$) \mathrm{b}$ & $0.05(-0.79$ to 0.88$)$ & $\mathrm{G} \times \mathrm{T}: 0.01$ \\
\hline
\end{tabular}

1RM bench press/BW (ratio)

\begin{tabular}{lllll}
\hline Before & $1.15 \pm 0.7$ & $1.06 \pm 0.6$ & $1.06 \pm 0.7$ & G: 0.34 \\
After & $1.23 \pm 0.8^{*}$ & $1.15 \pm 0.8^{*}$ & $1.07 \pm 0.7$ & T: 0.042 \\
ES & $0.11(-0.73$ to 0.94$)$ a & $0.12(-0.71$ to 0.96$)$ a & $0.01(-0.82$ to 0.85$)$ & G $\times$ T: 0.12 \\
\hline
\end{tabular}

1RM leg press [kg]

\begin{tabular}{lllll}
\hline Before & $239.7 \pm 52.6$ & $236.0 \pm 42.8$ & $244.5 \pm 35.8$ & G: 0.07 \\
After & $287.0 \pm 48.2 * * * \dagger$ & $277.5 \pm 53.2^{* \dagger}$ & $248.1 \pm 44.4$ & T: 0.001 \\
ES & $0.94(-0.02$ to 1.78$) \mathrm{c}$ & $0.86(-0.04$ to 1.7$) \mathrm{c}$ & $0.09(-0.75$ to 0.92$)$ & G $\times$ T: 0.009 \\
\hline
\end{tabular}

1RM leg press/BW (ratio)

\begin{tabular}{lllll}
\hline Before & $3.51 \pm 1.4$ & $3.55 \pm 1.5$ & $3.55 \pm 1.6$ & G: 0.92 \\
After & $4.13 \pm 1.7^{*}$ & $4.14 \pm 1.6^{*}$ & $3.62 \pm 1.7$ & T: 0.028 \\
ES & $0.38(-0.46$ to 1.23$) \mathrm{b}$ & $0.37(-0.48$ to 1.21$) \mathrm{b}$ & $0.04(-0.8$ to 0.88$)$ & G $\times$ T: 0.49
\end{tabular}


Table 2. Continued

\begin{tabular}{lllll}
\hline Variables & RT3 $(\mathrm{n}=11)$ & RT4 $(\mathrm{n}=11)$ & CG $(\mathrm{n}=11)$ & Significance $(\mathrm{p}$-values $)$ \\
\hline \multicolumn{2}{l}{ Bench press endurance $(\mathrm{reps})$} & & & \\
\hline Before & $21.7 \pm 2.3$ & $22.0 \pm 1.8$ & $21.1 \pm 3.4$ & G: 0.36 \\
After & $22.1 \pm 3.8$ & $21.6 \pm 3.0$ & $19.9 \pm 3.7$ & T: 0.42 \\
ES & $-0.13(-0.96$ to 0.71$) \mathrm{a}$ & $-0.16(-0.99$ to 0.68$) \mathrm{a}$ & $-0.34(-1.17$ to 0.52$)$ & $\mathrm{G} \times \mathrm{T}: 0.47$ \\
\hline Leg press endurance (reps) & & & \\
\hline Before & $24.2 \pm 4.1$ & $25.0 \pm 3.3$ & $25.3 \pm 4.6$ & G: 0.87 \\
After & $26.0 \pm 5.9$ & $25.9 \pm 4.3$ & $24.0 \pm 2.7$ & T: 0.66 \\
ES & $0.35(-0.5$ to 1.18$) \mathrm{b}$ & $0.23(-0.61$ to 1.06$) \mathrm{b}$ & $-0.34(-1.17$ to 0.51$)$ & G $\times$ T: 0.31 \\
\hline
\end{tabular}

RT3: RT 3 days per week, RT4: RT 4 days per week, CG: control group. BW: body weight. *denotes significant differences between before and after-training values $(\mathrm{p} \leq 0.05)$. ${ }^{* *}$ : denotes significant differences between the RT3 and RT4 groups at after-training $(\mathrm{p} \leq 0.05)$. $\dagger$ : denotes significant differences compared to $\mathrm{CG}(\mathrm{p} \leq 0.05) . \mathrm{G}=$ group, $\mathrm{T}=$ time. $\mathrm{a}, \mathrm{b}, \mathrm{c}$ denotes trivial, small, and moderate effect size (ES), respectively.

for 1RM bench press. Both the RT3 and RT4 groups showed a significant increase from before to after training and in comparison to the CG $(\mathrm{p}=0.025)$ with a moderate $(\mathrm{ES}=0.77)$ to small $(\mathrm{ES}=0.6) \mathrm{ES}$ in $1 \mathrm{RM}$ bench press. Post hoc analysis showed a significant $(\mathrm{p}=0.024)$ difference between training groups after the intervention, which indicated greater training effects for the RT3 group in 1RM bench press (Table 2).

There was a significant main effect of time $(\mathrm{F}=54.4$, $\mathrm{p}=0.001)$ and group $\times$ time $(\mathrm{F}=6.6, \mathrm{p}=0.009)$ interaction for 1RM leg press. Both the RT3 and RT4 groups showed a significant increase from before to after training and in comparison to the $\mathrm{CG}(\mathrm{p}=0.009)$ with a moderate $(\mathrm{ES}=0.94,0.86) \mathrm{ES}$ in 1RM leg press. Post hoc analysis showed a significant $(p=0.037)$ difference between training groups after the intervention, which indicated greater training effects for the RT3 group in 1RM leg press (Table 2).

There were no significant main effects of time $(\mathrm{F}=0.68$, $\mathrm{p}=0.42, \mathrm{~F}=0.20, \mathrm{p}=0.66)$ or group $\times$ time $(\mathrm{F}=0.75$, $\mathrm{p}=0.47, \mathrm{~F}=1.1, \mathrm{p}=0.31)$ interactions for upper - and lower-body muscular endurance, respectively. In addition, the RT3 and RT4 groups showed a trivial (ES $=-0.13$ and -0.16) ES in upper-body endurance, and a moderate $(\mathrm{ES}=0.35$ and 0.23$) \mathrm{ES}$ in lower-body endurance after the 8 -week training intervention (Table 2).

\section{Discussion}

The purpose of the current investigation was to examine the influence of 8-week RT with three sessions vs. four sessions per week under equated training volume on measures of muscular adaptations (i.e., thigh, arm, chest, and calf circumferences, percent body fat, 1RM of leg press and bench press, muscular endurance for the upper and lower body). The main findings from this study showed that both training modalities were effective to improve muscle size and muscular strength when controlled for training volume. In addition, three times RT during 8 weeks induced more adaptive responses in strength gains. The findings demonstrated that both RT groups improved body weight and decreased body fat; however, these findings were not statistically significant. In addition, the results from ESs were opposite (i.e., greater ES in body weight for the RT3 [ES, 0.31 vs. 011] and greater ES in body fat [ES, -0.5 vs. -0.18 ] for the RT4 group) (Table 2). In anthropometric measures, calf and arm circumferences did not statistically change after training for the RT3 and RT4 groups; however, the ES was greater for the RT4 compared with the RT3 group (i.e., small vs. trivial ES). In chest and thigh circumferences both the training groups showed significant increases from before to after training, whereas the RT3 group showed a greater in thigh (ES, 0.77 vs. 0.35$)$ and RT4 showed a greater change in chest (ES, 0.48 vs. 0.15 ) circumferences, respectively (Table 2). Changes in muscle size or CSA could be induced by increases in myofilaments, actin and myosin filaments, sarcoplasm, and connective tissue $[1,8,19]$. The findings of this study are in line with previous researchers who reported improvements in muscle size or hypertrophy after RT $[5,8,15,16]$. In addition, the results of this study are in line with previous reviews and meta-analyses that addressed the beneficial effects of RT on muscle hypertrophy [13, 23]. In relation to training frequency, Häkkkinen et al. [15] reported that two sessions per week of RT is more effective than one session per week to increase muscle CSA. Hoffman et al. [16] examined the effects of 10 weeks of different RT frequencies on muscular hypertrophy, and found that RT 4 and 5 times per week induced similar gains in chest and thigh circumferences. Recently, Schoenfeld et al. [23] in 
a systematic review and meta-analysis of 25 studies reported that "there is strong evidence that resistance training frequency does not significantly or meaningfully impact muscle hypertrophy when volume is equated," which is in line with our finding that gains in muscle size are not in relation to training frequency and training volume or dose response plays a crucial role to change in muscle size or hypertrophy, and more studies are necessary to explain the exact mechanism(s) for training frequency in muscle hypertrophy $[7,19,21]$.

The results demonstrated that both RT groups improved maximal strength after the 8-week intervention and both those training groups showed significant differences compared with the CG. In addition, the RT3 group showed significantly greater effects of training when compared with the RT4 group. To date, a large number of studies have reported that RT is an optimum training modality for strength gains in men and women $[1,3,5]$. Improving strength gains for the RT3 and RT4 groups when compared with the CG subjects who did not perform any training program explain the physiological effects of RT to enhance neuromuscular adaptations, such as improvements in inter-muscular coordination, increases in firing rate, and architecture mechanics in the single muscle fiber following the 8-week training period [3, 10]. However, strength gains after several weeks of training could be induced by enhancements in muscle size and hypertrophy $[1,8,19]$. In this study we did not make physiological assessments in relation to muscle hypertrophy (i.e., MRI), but we measured muscle circumference, body fat and body weight. Increases in muscle size with reduction of body fat and enhancements of body weight may explain the possible elevation of muscle hypertrophy, which was confirmed in previous meta-analysis studies.

Greater absolute strength gains in the RT3 group in comparison to RT4 may explain the dose response of strength gain after training intervention. However, when calculating relative changes in strength performance both the groups indicated trivial to small changes in 1RM bench press and leg press after the 8 -week training program. These findings may explain similar training benefits in strength performance when body weight is included. In fact, in absolute strength gains the RT3 group showed greater changes than the RT4 group and in body weight the RT3 group showed smaller changes than the trivial changes for the RT4, which explain the possibility of trivial to small strength in relation to body weight for both the training groups after the 8-week intervention. Other studies in men that compared training frequencies of (a) 1 vs. 2 per week, (b) 1 vs. 3 per week, and (c) 2 vs. 4 per week also showed similar gains in strength performance regardless of training frequency, which explains the greater role of training volume compared with training frequency $[4,6,7,12,15,16,20,22,25,26]$. On the other hand, Grgic et al. [14] reported that RT frequency plays a crucial role for increasing strength performance and higher RT frequencies induced greater strength gains. However, when training volume is equated, there was no significant effect of RT frequency on muscular strength gains, which is in line with our findings. In fact, adaptive responses in strength performance are in relation to training volume throughout the training period $[7,14]$ and more studies are necessary to explain the effects of RT frequency on gains in muscular strength.

Our results demonstrated no significant changes in upper- and lower-body muscular endurance for the RT3 and RT4 groups after the 8-week training intervention. These results are surprising, considering that improvements in muscular endurance with RT have been reported in some studies $[3,4]$. A possible explanation for these findings could be the large variability in responses among the participants in both groups. In addition, it seems that the training protocol used in this study was not sufficient to elicit significant increases in muscular endurance for the RT3 and RT4 groups, and more studies are needed to clarify the relationship between RT frequency and muscular endurance.

\section{Conclusions}

In summary, the results from this study indicated that 8 weeks of RT induced meaningful effects on strength performance adaptations and more RT days did not induce greater adaptive changes in muscular adaptations under volume-equated conditions. Both the RT regimes induced adaptive responses in muscular strength and anthropometric measures with greater training benefits for the RT3 group in strength gains.

Conflict of interest: Authors state no conflict of interest.

\section{References}

1. Abe T., DeHoyos D.V., Pollock M.L., Garzarella L. (2000) Time course for strength and muscle thickness changes following upper and lower body resistance training in men and women. Eur. J. Appl. Physiol., 81: 174-180.

2. American College of Sports Medicine. (2009) American College of Sports Medicine position stand. Progression models in resistance training for healthy adults. Med.Sci. Sports. Exerc., 41: 687-708.

3. Arazi H., Asadi A. (2011) Effects of 8 weeks equal-volume resistance training with different workout frequency on maximal strength, endurance and body composition. Int. J. Sports. Sci. Eng., 5(2): 112-118.

4. Arazi H., Asadi A., Roohi S. (2014) Enhancing muscular performance in women: compound versus complex, 
traditional resistance and plyometric training alone. J. Musc. Res., 17(2): 1450007, 1-10.

5. Arazi H., Damirchi A., Asadi A. (2013) Age-related muscle circumference, strength development and hormonal adaptations with 8 weeks moderate intensity resistance training. Annals. Endo., 74: 30-35.

6. Brigatto F.A., Braz T.V., Zanini T., Germano M.D., Aoki M.S., Schoenfeld B.J., Marchetti P.H., Lopes C.R. (2018) Effect of resistance training frequency on neuromuscular performance and muscle morphology after eight weeks in trained men. J. Strength. Cond. Res., 33(8): 2104-2116. DOI: $10.1519 /$ JSC.0000000000002563. Epub ahead of print.

7. Colquhoun R.J., Gai C.M., Aguilar D., Bove D., Dolan J., Vargas A., Couvillion K., Jenkins N.D.M., Campbell B.I., (2018) Training volume, not frequency, indicative of maximal strength adaptations to resistance training. $J$. Strength. Cond. Res, 32: 1207-1213.

8. Damas F., Phillips S., Vechin F.C., Ugrinowitsch C. (2015) A review of resistance training-induced changes in skeletal muscle protein synthesis and their contribution to hypertrophy. Sports. Med., 45: 801-807.

9. Dankel S.J., Mattocks K.T., Jessee M.B., Buckner S.L., Mouser J.G., Counts B.R., Laurentino G.C., Loenneke J.P. (2017) Frequency: The overlooked resistance training variable for inducing muscle hypertrophy? Sports Med., 47: 799-805.

10. Faul F., Erdfelder E., Lang A.G., Buchner A. (2007) $\mathrm{G}^{*}$ Power 3, a flexible statistical power analysis program for the social, behavioural and biomedical sciences. Behav. Res. Methods, 39: 175-191.

11. Fleck S.J., Kraemer W.J. (2004) Designing Resistance Training Programs, 3nd Ed. Champaign, IL: Human Kinetics.

12. Gomes G.K., Franco C.M., Nunes P.R.P., Orsatti F.L. (2019) High frequency resistance training is not more effective than low frequency resistance training in increasing muscle mass and strength in well-trained men. J. Strength Cond. Res., 33(Suppl 1): S130-S139. DOI: 10.1519/ JSC.0000000000002559.

13. Grgic J., Schoenfeld B.J., Davies T.B., Lazinica B., Krieger J.W., Pedisic Z. (2018) Effect of resistance training frequency on gains in muscular strength: A systematic review and meta-analysis. Sports Med., 48: 1207-1220.

14. Grgic J., Schoenfeld B.J., Latella C. (2018) Resistance training frequency and skeletal muscle hypertrophy: A review of available evidence. J. Sci. Med. Sport, 22(3): 361-370. DOI: 10.1016/j.jsams.2018.09.223.

15. Häkkinen K., Kallinen M. (1994) Distribution of strength training volume into one or two daily sessions and neuromuscular adaptations in female athletes. Elect. Clin. Neurophysiol., 34: 117-124.

16. Hoffman J.R., Kraemer W.J., Fry A.C., Deschenes M., Kemp D.M. (1990) The effect of self-selection for frequency of training in a winter conditioning program for football. J. Appl. Sport Sci. Res., 3: 76-82.

17. Hopkins W.G., Marshall S., Batterham A. (2009) Progressive statistics for studies in sports medicine and exercise science. Med. Sci. Sports Exerc., 41(1): 3-13.

18. Parrillo J., Greenwood-Robinson M. (1993) High-performance bodybuilding. Berkeley Publishing group, New York, 169-172.

19. Phillips S.M. (2014) A brief review of critical processes in exercise-induced muscular hypertrophy. Sports Med., 44: S71-S77.

20. Raastad T., Kirketeig A., Wolf D., Paulsen G. (2012) Powerlifters improved strength and muscular adaptations to a greater extent when equal total training volume was divided into 6 compared to 3 training sessions per week. 17th Annual Conference of the ECSS, Brugge, Belgium, July 4-7.

21. Ralston G.W., Kilgore L., Wyatt F.B., Baker J.S. (2017) The effect of weekly set volume on strength gain: A meta-analysis. Sports Med., 47: 2585-2601.

22. Saric J., Lisica D., Orlic I., Grgic J., Krieger J.W., Vuk S., Schoenfeld B.J. (2018) Resistance training frequencies of 3 and 6 times per week produce similar muscular adaptations in resistance-trained men. J. Strength Cond. Res., 33(Suppl 1): S122-S129. DOI: 10.1519/ JSC.0000000000002909.

23. Schoenfeld B.J., Grgic J., Krieger J. (2018) How many times per week should a muscle be trained to maximize muscle hypertrophy? A systematic review and meta-analysis of studies examining the effects of resistance training frequency. J. Sports Sci., 37(11): 1286-1295. DOI: 10.1080/02640414.2018.1555906.

24. Schoenfeld B.J., Ogborn D., Krieger J.W. (2016) Effects of resistance training frequency on measures of muscle hypertrophy: A systematic review and meta-analysis. Sports Med., 46: 1689-1697.

25. Schoenfeld B.J., Ratamess N.A., Peterson M.D., Contreras B., Tiryaki-Sonmez G. (2015) Influence of resistance training frequency on muscular adaptations in welltrained men. J. Strength Cond. Res., 29: 1821-1829.

26. Yue F.L., Karsten B., Larumbe-Zabala E., Seijo M., Naclerio F. (2018) Comparison of 2 weekly-equalized volume resistance-training routines using different frequencies on body composition and performance in trained males. Appl. Physiol. Nutr. Metab., 43: 475-481.

\section{Received 09.11.2019 \\ Accepted 09.05.2020}

(C) University of Physical Education, Warsaw, Poland

\section{Acknowledgments}

The authors are thankful to the participants of this study for their excellent collaborations. 